\title{
Nuevos registros de peces costeros tropicales para el Perú
}

\section{New records of coastal tropical fish in Peru}

Yuri Hooker M.

Laboratorio de Biología Marina, Facultad de Ciencias Biológicas y Fisiológicas, Universidad Peruana Cayetano Heredia. Calle Honorio Delgado 430, SMP. Lima, Perú. E-mail: hookery@yahoo.com

Presentado: $15 / 05 / 2009$ Aceptado: 27/06/2009 Publicado online: $28 / 08 / 2009$

\section{Resumen}

En el presente trabajo se dan a conocer 11 nuevos registros de peces marinos para el Perú. Las colectas fueron efectuadas entre los años 1989 y 2007 en las localidades de Bocapan, Canoas de Punta Sal, Punta Sal (Tumbes); Los Órganos, Isla Foca (Piura); Islas Lobos de Afuera (Lambayeque); Bahía Samanco (Ancash); y Pucusana (Lima). Los nuevos registros para la ictiofauna marina del Perú son: Dasyatis longa, Urobatis halleri, Cephalopholis panamensis, Prognathodes carlhubbsi, Cirrhitus rivulatus, Stegastes beebei, Thalassoma lucasanum, Thalassoma grammaticum, Axoclinus lucillae, Elacatinus punticulatus y Coryphopterus urospilus. Las especies Cirrhitichthys oxycephalus, Lythrypnus dalli y Myripristis leiognathus, fueron anteriormente mencionadas para Perú pero sin existir registros documentados, con la presente publicación se certifica su presencia. Se discute sobre características biogeográficas y ampliación de distribución durante El Niño.

Palabras clave: Nuevos registros, peces tropicales, biodiversidad marina, Provincia biogeográfica Panámica, El Niño.

\section{Abstract}

In this paper I report on 11 new records of marine fishes from Peru. The collections were made between 1989 and 2007 in Bocapan, Canoas de Punta Sal, Punta Sal (Tumbes); Los Órganos, Isla Foca (Piura); Islas Lobos de Afuera (Lambayeque); Bahía Samanco (Ancash); y Pucusana (Lima). The new records for the marine fish fauna of Peru are: Dasyatis longa, Urobatis halleri, Cephalopholis panamensis, Prognathodes carlhubbsi, Cirrhitus rivulatus, Stegastes beebei, Thalassoma lucasanum, Thalassoma grammaticum, Axoclinus lucillae, Elacatinus punticulatus and Coryphopterus urospilus. Cirrhitichthys oxycephalus, Lythrypnus dalli and Myripristis leiognathus, previously have been referred to Peru but not documented there, this paper certify their presence

Keywords: New records, tropical fishes, marine biodiversity, Panamic biogeographic province, ENSO.

\section{Introducción}

La ictiofauna marina del Perú es bien conocida y es presentada en numerosas publicaciones entre las que destacan Chirichigno (1962, 1963a, 1963b, 1969, 1973, 1978, 1987); Chirichigno y Iwamoto (1977), Chirichigno y McEachran (1979), Chirichigno y Vélez (1998); Chirichigno y Cornejo (2001), Hooker (1990, 1993, 2000), Vildoso et al. (1999), entre otros. La mayoría de registros han sido obtenidos de pesquerías artesanales y en cruceros de investigación del IMARPE, así como el aporte personal de algunos investigadores o colaboradores independientes. Sin embargo, aun existen vacíos de conocimiento sobre áreas poco estudiadas o de difícil acceso (Vildoso et al. 1999), que podrían albergar nuevas especies de peces marinos.

La diversidad del litoral peruano al norte de los 4º15'S, está caracterizada por fauna representativa de la Provincia biogeográfica Panámica, encontramos aqui alrededor del $70 \%$ de todas las especies asociadas a los arrecifes rocosos del litoral peruano (Hooker 1993); algunas familias de peces tropicales como Labridae, Chaetodontidae, Cirrhitidae, Gobiidae están bien representadas en esta área. Muchas de las especies suelen ser pequeñas, crípticas, vivir en grietas entre las rocas, usar escondites estrechos de los cuales normalmente no se alejan y en algunos casos, además son especies raras, con poblaciones de muy baja abundancia y por tanto difíciles de encontrar o capturar.

El presente trabajo se describe y reportan 11 especies de peces, incrementando el conocimiento sobre la biodiversidad marina del Perú, en especial de las aguas costeras tropicales poco profundas.

\section{Material y métodos}

Las colectas y registros se realizaron entre los ańos 1985 y 2007. La captura de los especimenes fue directa, por medio de buceo en apnea, buceo SCUBA y buceo con compresora hookah, utilizándose arpones, así como redecillas de mano para los especimenes más pequeños. La mayoría de las especies fueron fotografiadas in situ por medio de cámaras fotográficas submarinas Nikonos V y cámaras digitales con caja estanca. Todas las fotos presentadas fueron realizadas por el autor. Posteriormente, los especimenes colectados fueron fotografiados muertos, registrando el lado izquierdo del cuerpo. Las muestras fueron fijadas en formol al 10\% y preservadas en colección en alcohol al 70\%. Para la revisión taxonómica se siguió a Allen y Robertson (1994), Compagno (1999), Fischer et al. (1995), Humann y Deloach (1993), Meek y Hildebrand (1928) y Robertson y Allen (2002). Para la clasificación se siguió a Nelson (1994). Las medidas obtenidas se presentan en milímetros y las abreviaturas de las partes anatómicas se dan según Chirichigno y Vélez (1998). Los especímenes colectados se encuentran depositados en la Colección de Zoológia Acuática (CZA) del Laboratorio de Biología Marina de la Universidad Peruana Cayetano Heredia y en la colección del Instituto del Mar del Perú (IMARPE).

Los nombres comunes que se mencionan son los nombres locales, algunas especies no son conocidas por los pescadores.

\section{Resultados}

Phylum: Chordata

Clase: Chondrichthyes

ORDEN: RAJIFORMES

FAMILIA: DASYATIDAE

\section{Dasyatis longus (Garman, 1880)}

\section{Raya látigo}

Material examinado: Dos especímenes hembras $(1640 \mathrm{~mm}$ LT, AD $750 \mathrm{~mm}$ y $2080 \mathrm{~mm}$ LT, AD: $960 \mathrm{~mm}$ ), colectado por pescadores artesanales en Bocapan, Tumbes (03\%1'17"S; $80^{\circ} 41$ ' 42 ”W), el 02 de agosto de 2001. Solo se conservaron las mandíbulas (IMARPE $\mathrm{s} / \mathrm{n}$ ). 
Registros adicionales: En 1981 se observó bajo el agua a un espécimen de aproximadamente $1500 \mathrm{~mm}$ de ancho de disco (AD) (calculado bajo el agua por extensión de brazos del autor) en Punta Sal, Tumbes (0358'59,7'S, 8059'12,7”W). Hasta 1990 era una especie frecuente en la zona entre Punta Sal y Punta Mero, observándose varios especímenes a profundidades menores de $5 \mathrm{~m}$. A. Nakandajari y $\mathrm{S}$. Gonzales registran un espécimen de D. longus (2560 mm LT, $1230 \mathrm{~mm} \mathrm{AD)} \mathrm{desembarcado} \mathrm{en}$ Puerto Pizarro, Tumbes el 19 de enero del 2008. La mandíbula está conservada en la colección del Laboratorio de Selacología y Arqueozoología de la Universidad Peruana Cayetano Heredia.

Caracteres de diagnostico: Disco ovalado, más ancho que largo; márgenes anteriores rectos; cola delgada, más del doble del largo del disco; dorsalmente una fila media de espinas romas, desde la cabeza hasta cerca de la cintura pectoral. Cola con una quilla dorsal y un pliegue ventral largo y bajo. Encontramos que la longitud interorbital es de alrededor de 6 veces en el ancho del disco mientras que en $D$. brevis es aproximadamente de 5,2 en $\mathrm{AD}$.

Observaciones: Actualmente es una especie rara en las costas de Tumbes, siendo habitualmente capturada por buzos artesanales y pescadores con redes. En los años 1980 era frecuente y poco capturada por su bajo precio, sin embargo en la actualidad es muy buscada lo que aparentemente la ha llevado a ser sumamente rara. Especie de hábitos costeros, que permanece inmóvil durante el día, semienterrada en la arena, siempre cerca de los arrecifes rocosos. La especie se encuentra dentro de la Lista Roja de especies amenazadas de la IUCN con la categoría DD (Data Deficiente), justificándose su inclusión además por su gran tamaño y la baja fecundidad (1 a 5 crías por parición), lo que sugiere que la especie podría ser altamente vulnerable a las pesquerías, aspecto poco conocido (Smith 2006). Nuestras observaciones justificarían que sea incluida dentro de la lista de especies amenazadas del Perú.

Distribución: Conocida desde Ometepec, en el extremo norte del Golfo de California a Puerto López, Ecuador, incluyendo las Islas Galápagos (Robertson y Allen 2002). Con el presente reporte se amplia hasta Punta Sal, Tumbes, Perú.

\section{FAMILIA:UROLOPHIDAE}

\section{Urobatis halleri (Cooper, 1863)}

\section{Raya Batea (Fig. 1)}

Material examinado: Un espécimen hembra de $470 \mathrm{~mm} \mathrm{LT}$, AD 268 mm, colectado en Los Órganos, Piura (04¹0'38” S; $81^{\circ} 08^{\prime} 36^{\prime \prime}$ W) a $4 \mathrm{~m}$ de profundidad, en febrero de 1989 . Solo se conservó las mandíbulas (IMARPE s/n).

Registros adicionales: También ha sido observada y fotografiada en Islas Lobos de Afuera (0656'01,2”S; 8042'19,9”W), en marzo de 2000 y en Canoas de Punta Sal (03056'51"S; $\left.80^{\circ} 56^{\prime} 50^{\prime \prime} \mathrm{W}\right)$, en diciembre de 2007 y febrero de 2008.

Caracteres de diagnostico: Disco redondo, ligeramente más largo que ancho, con las márgenes anteriores rectas; cola igual o menor que la mitad de la longitud total; caudal redondeada; con 26 a 35 filas de dientes en la mandíbula superior (Allen y Robertson 1994). Coloración peculiar de carácter diagnóstico: disco color crema, con grandes manchas circulares marrones

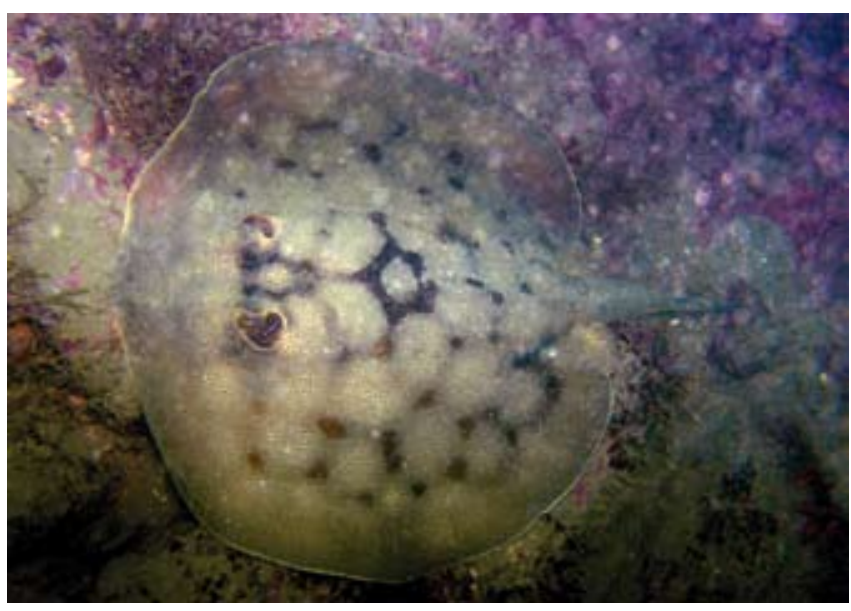

Figura 1. Urobatis halleri, Canoas de Punta Sal, Tumbes. Diciembre de 2007.

oceladas que le dan aspecto reticular, a su vez, todo el disco cubierto por pequeñas manchas marrón oscuro (no evidentes en el espécimen de Lobos de Afuera). Una delgada línea blanquizca bordea el margen del disco.

Observaciones: Es una especie frecuente en aguas poco profundas de las costas de Tumbes. En Lobos de Afuera solo fue observada en una ocasión. Todos los especimenes han sido observados semienterrados en la arena entre los arrecifes rocosos o en pequeńas cuevas rocosas con fondo arenoso.

Distribución: De Elkhorn Slough, Monterey Bay, California (Eschmeyer, 1998) hasta Ecuador (Béarez, 1996). Con el presente hallazgo se amplía hasta Los Órganos, Perú, incluyendo las Islas Lobos de Afuera.

Clase: ActinopterygiI

Orden: Bericiformes

Familia: Holocentridae

\section{Myripristis leiognathus Valenciennes, 1846}

(Fig. 2)

Material examinado: Dos especimenes de 68 y $87 \mathrm{~mm}$ (IMARPE s/n), Bahía Samanco, Ancash (9¹2’12,25”S, $78^{\circ} 33^{\prime} 22,7^{\prime \prime W)}$ el 30 de agosto de 1997. Dos especimenes de 104 y 119 mm (CZA-9), Bahía Samanco, Ancash (9¹2’12,25”

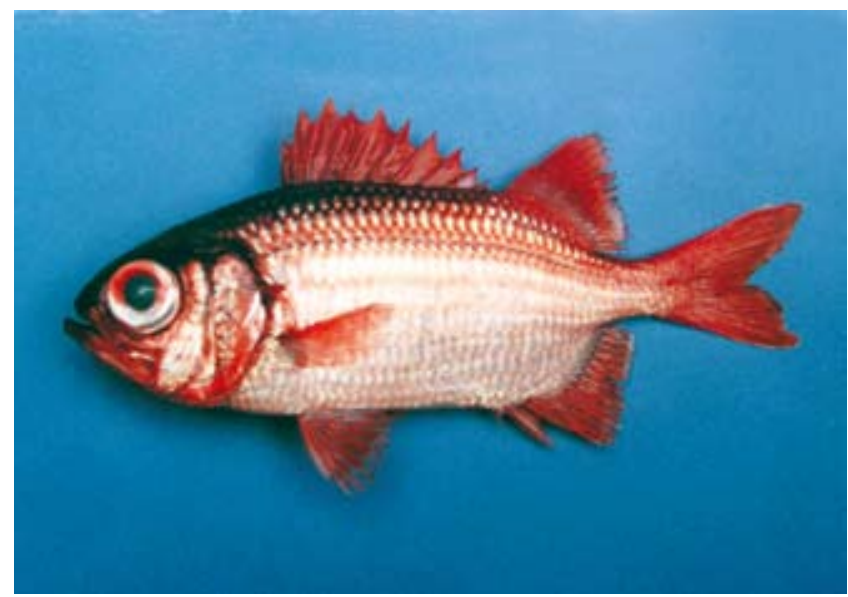

Figura 2. Myripristis leiognathus, Bahía Samanco, Ancash. Agosto de 1997. 
S, 78³3'22,7”W) el 30 de agosto de 1997. Un espécimen de 62 mm LS (CZA-10), Los Órganos, Piura (04¹0'38”'S; $\left.81^{\circ} 08^{\prime} 36^{\prime} \mathrm{W}\right)$ el 11 de marzo de 2004.

Caracteres de diagnostico: D: X, I, 13-15; A: IV, 11-13. Ojos grandes (2,2 a 2,3 veces en la cabeza en nuestros especímenes) y espinas prominentes en las aletas. Escamas grandes y ásperas, 2,5 hileras escamas sobre la línea lateral; línea lateral con 34 a 40 escamas; branquiespinas 28-34; axila pectoral sin escamas (Robertson y Allen 2002). Coloración totalmente roja con los bordes de las escamas más oscuros; ojo con una barra vertical negra que al morir se pierde rápidamente.

Observaciones: Peces poco frecuentes en el área de distribución (encontrado entre Canoas de Punta Sal, Tumbes y Los Órganos, Piura). Durante el día permanecen ocultos en estrechas y profundas cavidades de las rocas. Por la noche se les encuentra nadando libremente en los espacios próximos a las rocas, en parejas o pequeños grupos, capturando organismos planctónicos individuales. Durante El Niño 1997-98 fue frecuente en bahía Samanco donde solo se observaron juveniles. En años normales no fue observado en esta localidad.

Distribución: Golfo de California a Ecuador, incluyendo las islas Galápagos. Mencionado por Chirichigno y Cornejo (2001), se confirma aquí su distribución hasta Los Órganos, Perú. Durante El Niño alcanza bahía Samanco, Perú.

Orden: Perciformes

FAMILIA: SERRANIDAE

\section{Cephalopholis panamensis (Steindachner, 1877)}

\section{Mero de PeŃa (Fig. 3)}

Material examinado: Dos especimenes de 138 y $240 \mathrm{~mm}$ LS (IMARPE s/n), colectados en Los Órganos, Piura (04 $10^{\prime} 38^{\prime \prime}$ S; $81^{\circ} 08^{\prime} 36^{\prime}$ W) a $5 \mathrm{~m}$ de profundidad, en mayo de 1989 . Un espécimen de 294 mm LS (CZA-01), colectado en Los Órganos, Piura, en febrero de 1990.

Registros adicionales: En junio de 1999 y noviembre del 2007 se observó varios especimenes en las islas Lobos de Afuera.

Caracteres de diagnostico: D: IX, 14; A: III, 8; P: 17- 18; branquiespinas $16-19$. Aleta caudal redondeada; escamas

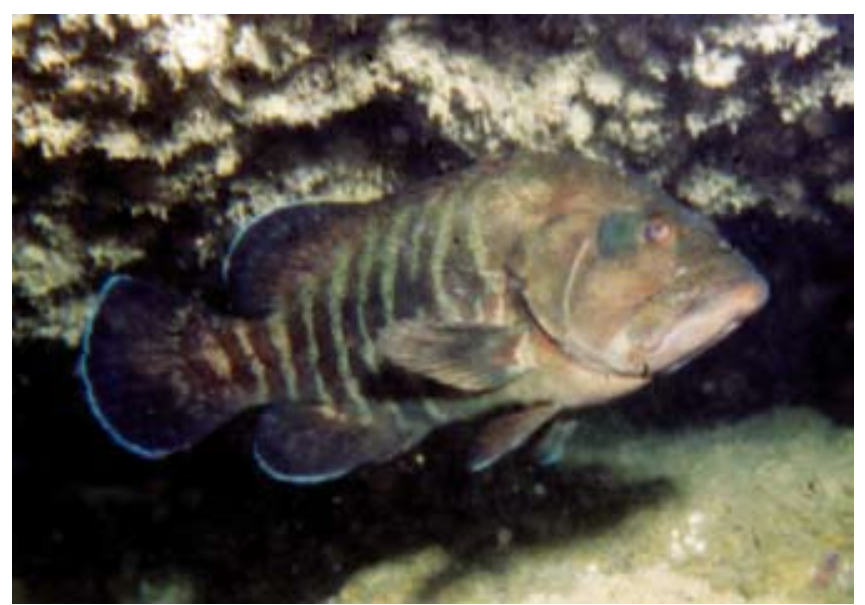

Figura 3. Cephalopholis panamensis, islas Lobos de Afuera. Junio de 1999 . del flanco ásperas (Allen y Robertson 1994, Robertson y Allen 2002). Cuerpo con barras alternadas gris claro y marrón oscuro; cabeza con puntos anaranjados; con una mancha post ocular azulada; aletas dorsal, anal y caudal con borde celeste claro.

Observaciones: es una especie frecuente en la zona, pero difícil de observar pues durante el día permanece oculta dentro de cuevas estrechas. Es ocasionalmente capturado por buzos artesanales.

Distribución: Golfo de California (Heemstra y Randall, 1993) a Ecuador (Béarez 1996), incluyendo las islas Galápagos (Humann y Deloach, 1993). Con el presente hallazgo se amplia su distribución hasta Los Órganos, Perú, incluyendo las islas Lobos de Afuera.

\section{Familia: Chaetodontidae}

\section{Prognathodes carlhubbsi Nalbant, 1995}

(Fig. 4)

Material examinado: Un espécimen de $121 \mathrm{~mm} \mathrm{LS}$ (CZA-02) colectado en las islas Lobos de Afuera (0656'29”S; $80^{\circ} 43^{\prime} 51$ ”W) a $35 \mathrm{~m}$ de profundidad, el 27 marzo de 2000.

Registros adicionales: No se ha registrado otro espécimen.

Caracteres de diagnostico: D: XIII, 19- 20; A: III, 1416; P: 13- 14. Línea lateral con 37 a 49 escamas (Robertson y Allen 2002). Espinas dorsales muy alargadas; segunda espina anal muy alargada; hocico alargado, tubular. Color del cuerpo amarillo, con una mancha distintiva negra, en forma de guadaña o "V" invertida que alcanza la base de las espinas dorsales; también una barra negra desde el hocico a la primera espina de la dorsal, cubriendo el ojo. Base de la aleta dorsal marrón grisácea. Espinas y primer radio de la aleta ventral amarillos, todos los otros radios enteramente negros.

Observaciones: Robertson y Allen (2002) mencionan que $P$. carlhubbsi es una especie muy similar a $P$. falcifer, la cual está distribuida al norte de la región tropical de Pacifico Este (Sur de California, golfo de California e islas Revillagigedo). Ambas especies no tienen diferencias morfométricas o merísticas significativas, siendo diferenciadas solamente por su coloración y su distribución. P. calcifer se diferencia de nuestra especie por no presentar la barra negra sobre el hocico (solo es ligeramente

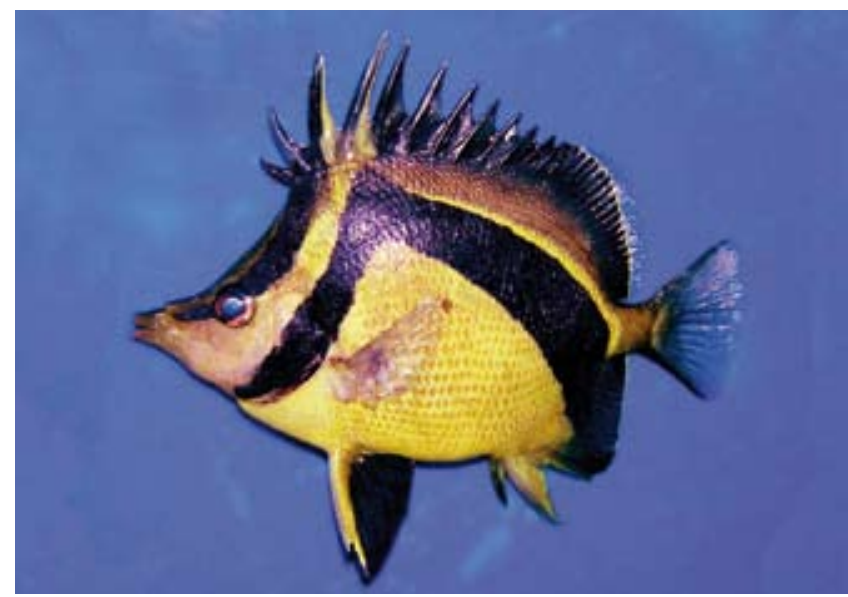

Figura 4. Prognathodes carlhubbsi, islas Lobos de Afuera. Marzo del 2000 
grisácea), la mancha en forma de guadaña no alcanza la base de la dorsal y la base de la aleta dorsal es amarilla. Adicionalmente nosotros observamos que en $P$. calcifer los radios de la aleta ventral son amarillos con el borde negruzco y que los lados del cuerpo por debajo de la mancha en guadańa son blanquizcos.

El espécimen fue capturado a $35 \mathrm{~m}$ de profundidad, dentro de una pequeña cueva de un arrecife rocoso con aguas turbulentas. Este es el único espécimen que ha sido observado durante los años de estudio. Se presume sea una especie que llega ocasionalmente al Perú o que vive a profundidades no alcanzables por buceo SCUBA. Robertson y Allen (2002) mencionan que vive entre los 12 y $270 \mathrm{~m}$.

Distribución: Solo conocida en las Islas Galápagos, Islas del Coco y Malpelo. (Robertson y Allen 2002). Se amplia su distribución hasta las Islas Lobos de Afuera, Perú.

FAMILIA: Cirrhitidae

\section{Cirrhitus rivulatus Valenciennes, 1846}

\section{Mero MAPA (Fig. 5)}

Material examinado: Un espécimen de $242 \mathrm{~mm}$ LS (IMAR-

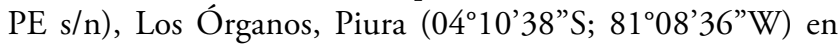
mayo de 1989. Un espécimen de $347 \mathrm{~mm}$ LS (IMARPE s/n), en la isla Foca, Piura $\left(05^{\circ} 12^{\prime} 07^{\prime \prime}\right.$; ; 81 $\left.12^{\prime} 29^{\prime \prime W}\right)$ el 28 de agosto de 1998.

Registros adicionales: En 1989 se colectaron 2 especímenes, uno de ellos fue analizado internamente y el otro conservado en alcohol para colección. En junio de 1999 se observó 3 especímenes en las islas Lobos de Afuera (06 $55^{\prime} 09^{\prime}$ 'S; 8044’09”W).

Caracteres de diagnostico: D: X, 11- 12; A: III, 6. Un penacho de cirros cerca del extremo de cada espina dorsal; radios pectorales inferiores con las puntas libres; preoperculo finamente aserrado. Coloración conspicua, cuerpo marrón claro, con manchas irregulares formando barras, de color anaranjado bordeadas de una línea negra, la que a la vez está marginada por una línea celeste; aleta caudal con coloración reticulada celeste.

Observaciones: Un pez frecuente en zonas rocosas del litoral de Piura y Tumbes. En las islas Lobo de Afuera es una especie rara vez vista. Es una especie difícil de observar por permanecer oculta en grietas y pequeńas cuevas desde donde observa el exterior. Se analizó el contenido estomacal de un espécimen

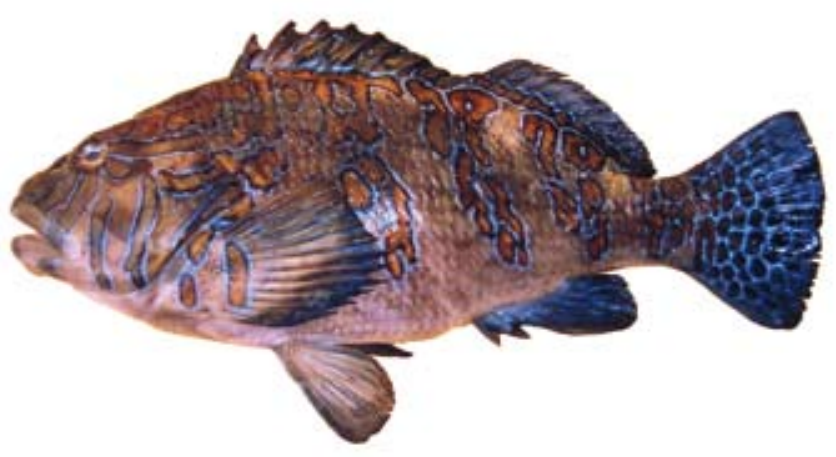

Figura 5. Cirrhitus rivulatus, isla Foca, Piura. Agosto de 1998. colectado en 1989 en Los Órganos, encontrándose abundantes restos de pequeños cangrejos de la familia Majidae.

Distribución: Del Golfo de California hasta Ecuador, incluyendo todas las islas oceánicas hasta Galápagos (Robertson y Allen 2002). Se amplia su distribución hasta Los Órganos, Piura incluyendo la isla Foca y las islas Lobos de Afuera. Se desconoce si su presencia en isla Foca se debe a una extensión costera de su distribución a causa de El Niño.

\section{Cirrhitichthys oxycephalus (Bleeker, 1855)}

(Fig. 6)

Material examinado: Un espécimen de $83 \mathrm{~mm}$ LS (IMARPE $s / n$ ), en la plataforma petrolera de Los Órganos, Piura (409'51,8”S; 8110’01,6”W) el 08 de enero de 1999.

Registros adicionales: No se ha registrado otro espécimen.

Caracteres de diagnostico: D: X, 12; A: III, 6; pectorales con los 6 radios inferiores con puntas libres, sin ramificar; el primer radio blando de la dorsal suave y prolongado; un penacho de cirros cerca del extremo de cada espina dorsal; preoperculo fuertemente aserrado. Color rosado claro, con manchas rojo oscuro; cabeza con manchas más pequeñas marrón rojizo o rojas; primer radio dorsal y radio superior de la caudal amarillentos. Región ventral blanquizca.

Observaciones: En el Perú solo se ha registrado un espécimen durante todo el tiempo de estudio, el cual fue hallado oculto entre ascidias coloniales, sobre la estructura metálica de la plataforma petrolera de Los Órganos, a $8 \mathrm{~m}$ de profundidad.

Distribución: Del Golfo de California al norte de Perú (Chirichigno y Cornejo 2001), incluyendo todas las islas oceánicas. También presente en el Indopacífico (Robertson y Allen 2002). Se confirma su distribución hasta Los Órganos, Perú.

FAMilia: Pomacentridae

\section{Stegastes beebei (Nichols, 1924)}

(Fig. 7)

Material examinado: Un espécimen juvenil de $37 \mathrm{~mm}$ LS (CZA-03), Bahía Samanco, Ancash (09¹2’04”S; 78³3’27”W) el 8 de agosto de 1997.

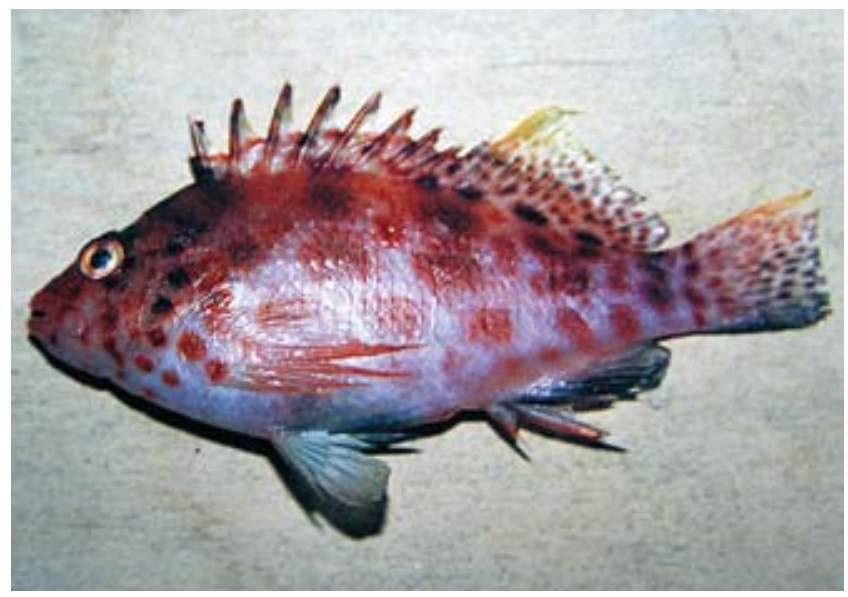

Figura 6. Cirrhitichthys oxycephalus, Los Órganos, Piura. Enero de 1999. 


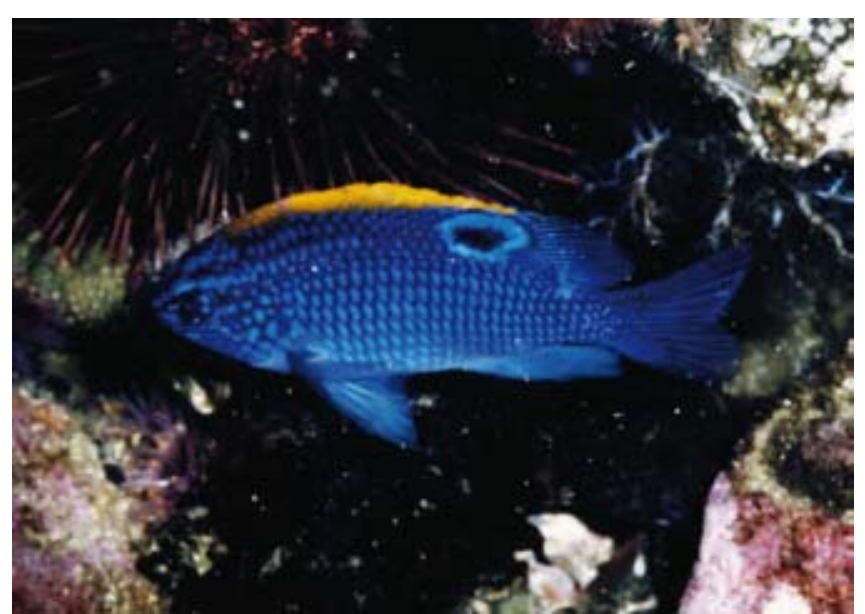

Figura 7. Stegastes beebei, isla Foca, Piura. Setiembre de 1997.

Registros adicionales: En setiembre de 1997 (El Niño), en isla Foca, Piura, se detectaron 2 especímenes juveniles de menos de $3 \mathrm{~cm}$. Durante el verano de 1998 (El Nińo) varios juveniles fueron observados en Pucusana, Lima (12²8'36"S, $76^{\circ} 47^{\prime} 52^{\prime} \mathrm{W}$ ) y en varios lugares dentro de la bahía Samanco. En junio de 1999 se observaron 3 especímenes adultos en las islas Lobos de Afuera (0656'30”S; 8043'21”W), uno de ellos registrado en video (disponible en http://www.youtube.com/ watch?v=j9cZZlIVc1Y). En octubre de 2007 se observaron dos individuos adultos en la isla Lobos de Afuera, sector Los Lagartos.

Caracteres de diagnostico: D: XII, 14- 16 (15 en espécimen CZA-03); A: II, 13, raramente 14 (13 en CZA-03); P: 20- 22 (21 en CZA-03); 20 escamas en la línea lateral (raramente 19); 10 - 12 espinas branquiales en la rama inferior del 1er arco branquial (10 en CZA-03) (Allen y Robertson 1994, Fischer et al. 1995). En vivo el adulto con patrón de coloración distintivo: cuerpo marrón oscuro; una banda blanca en el pedúnculo caudal; parte inferior del ojo azul; ángulo exterior de la pectoral blanquizco, con una mancha amarilla en la punta de la aleta. Juvenil azul oscuro iridiscente, con la nuca, dorso y espina dorsales rojas o naranjas y un ocelo azul con centro negro en la base posterior de la aleta dorsal. Los juveniles pequeños observados en Perú no presentan la banda blanca sobre el pedúnculo, como si ocurre en los especímenes citados por Allen y Robertson (1994), posiblemente por ser especímenes muy pequeños. Se le

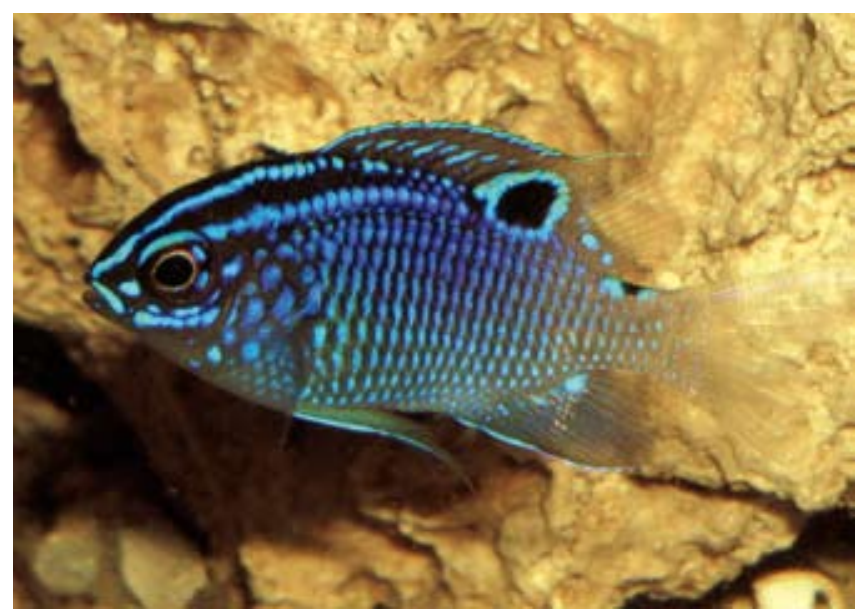

Figura 8. Stegastes acapulcoensis, isla Foca, Piura. Junio del 2009. puede confundir con los juveniles de S. acapulcoensis (Fig. 8), sin embargo estos no presentan el dorso anaranjado.

Observaciones: Especie poco abundante, solo se ha observado adultos en las islas Lobos de Afuera. Durante el fenómeno de El Niño 87-98' los juveniles fueron muy comunes en isla Foca (Piura), bahía Samanco, bahía Guaynumá (Ancash) y en Pucusana (Lima). En años normales no se les ha observado en el litoral continental. Los adultos observados en Lobos de Afuera se mostraban territoriales no dejando que otros peces ingresen a las proximidades de su refugio.

Distribución: Conocido en islas oceánicas (Malpelo, Cocos y Galápagos). Raros en la costa de Costa Rica y Panamá (Robertson y Allen 2002). Se amplía su distribución hasta Islas Lobos de Afuera, Perú. Durante El Niño amplia su distribución hasta Pucusana.

\section{FAMILIA: LABRIDAE}

\section{Thalassoma lucasanum (Gill, 1862)}

$$
\text { Viejita Cabeza AZUl (Figs. } 9 \text { y } 10 \text { ) }
$$

Material examinado: Tres especímenes de 118, 120 y 142 mm LS (IMARPE s/n), Los Órganos, Piura (04¹0’38”s; 8108'36”W) en mayo de 1989.

Registros adicionales: Común en las costas rocosas entre

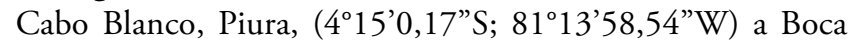
Pan, Tumbes. También registrada en las islas Lobos de Afuera (06 54'52,5”S; 8042'55,9”W) en Marzo del 2000 y octubre del 2007. Durante el verano de 1998 (El Niño), varios juveniles fueron observados en bahía Samanco (09¹2’04”S; 78³3’27”W), Ancash y en Pucusana, Lima.

Caracteres de diagnostico: Características morfométricas y merísticas no representativas, pudiendo confundirse con otras especies de la familia. Coloración diagnóstica. Especie hermafrodita protogínica (cambian de sexo de hembras a machos) con coloración distintiva: Fase inicial (juveniles y hembras) presenta una línea negra a lo largo del dorso, lateralmente con una secuencia de líneas amarilla, negra, amarilla y roja, atravesando longitudinalmente el cuerpo; vientre blanco. Fase terminal (hembra transformada en macho) con cabeza azul y cuerpo lila con borde de las escamas delineadas en azul; una mancha vertical

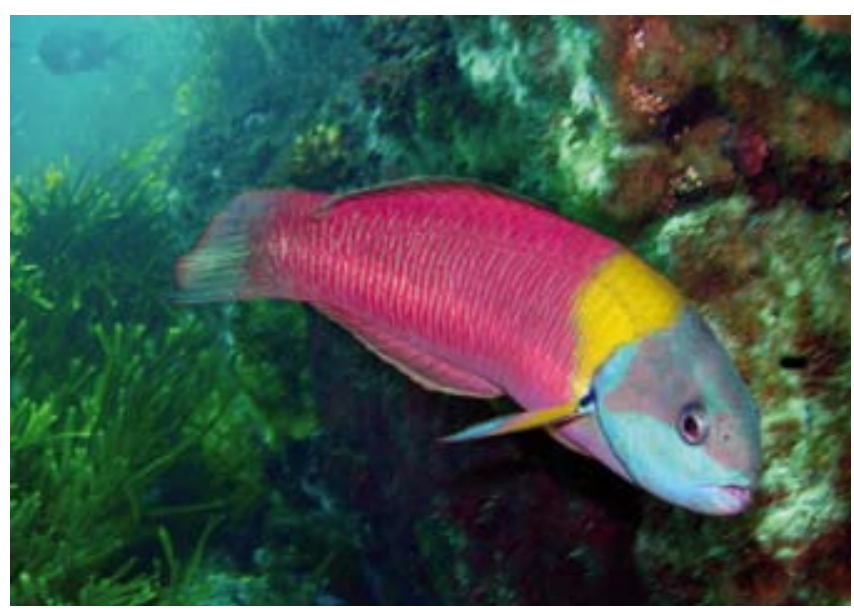

Figura 9. Thalassoma lucasanum, adulto en fase terminal. Isla Lobos de Afuera. Octubre del 2007. 


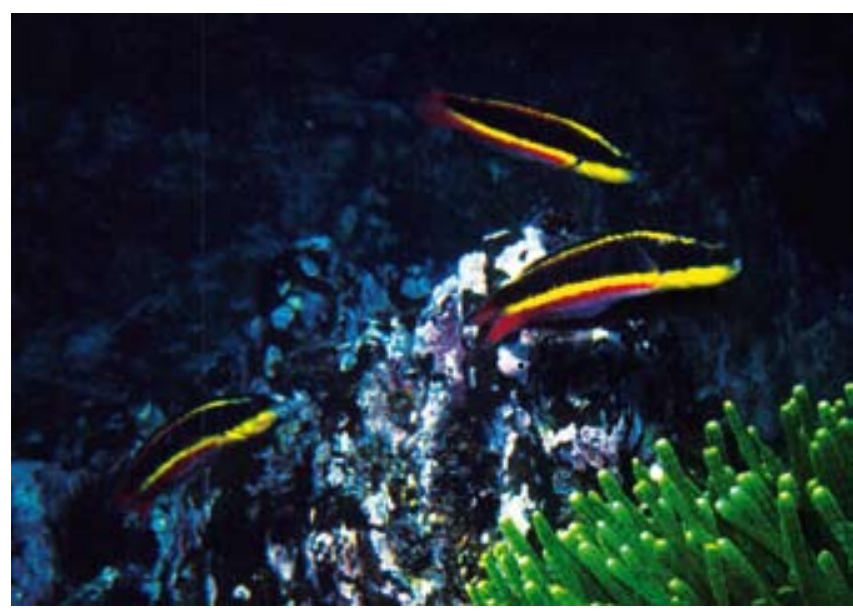

Figura 10. Thalassoma lucasanum, juveniles en fase inicial. Isla Lobos de Afuera. Marzo del 2000.

postcefálica amarilla; pectorales amarillas con el extremo azul; radios de la caudal azules.

Observaciones: Especie merodeadora con sociedades tipo harem, conformado por uno o dos machos y un número superior a 5 hembras y/o juveniles. También es frecuente encontrar hembras con cambio parcial de coloración. Según lo observado, cada harem estaría dominando un conjunto rocoso, manteniendo distancia considerable de otros grupos. En observaciones de campo y acuario se vio que, al igual que otras especies de lábridos, T. lucasanum se oculta bajo la arena durante la noche. La especie era muy común en su área de distribución pero en los últimos 5 años ha disminuido notoriamente por ser capturado y comercializado como especie de acuario.

Distribución: Golfo de California a Ecuador, incluyendo las islas Galápagos (Robertson y Allen 2002). Amplia su distribución hasta Cabo Blanco, Perú, incluyendo las islas Lobos de Afuera. Durante El Niño, llega hasta Pucusana, Lima.

\section{Thalassoma grammaticum Gilbert, 1890}

(Fig. 11)

Material examinado: Un espécimen de $133 \mathrm{~mm}$ LS (CZA4), isla Lobos de Afuera (0654'52,5”'S; 8042'55,9”W) el 28 de marzo de 2000

Registros adicionales: En el lugar de colecta se observaron 4 especímenes. No se conoce de registro en otro lugar del litoral peruano.

Caracteres de diagnostico: D: VIII, 13- 14; A: III, 11; P: 15- 17 (generalmente 16); línea lateral con 25 escamas con

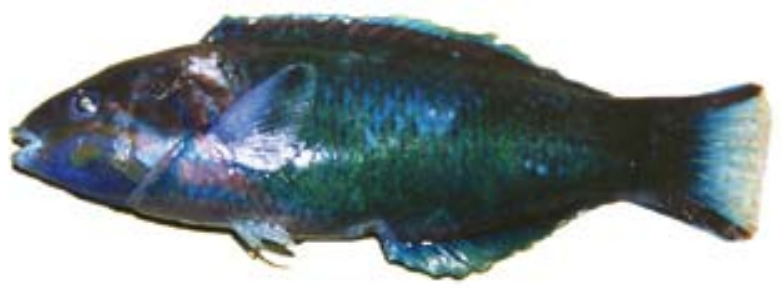

Figura 11. Thalassoma grammaticum, isla Lobos de Afuera. Marzo de 2000.

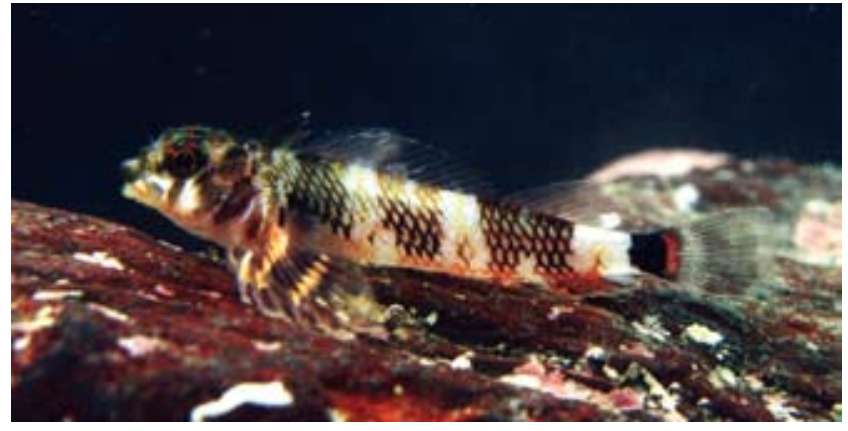

Figura 12. Axoclinus lucillae, isla Foca, Piura. Mayo de 1999.

poros (Robertson y Allen 2002). Especie hermafrodita protogínica con coloración distintiva: Fase terminal con cuerpo verde azulado, cabeza azul verdosa con franjas lila en la mejilla y parte anterior del abdomen; aleta pectoral celeste; radios marginales de la caudal lilas; base de la dorsal y anal, lila.

Observaciones: Especie muy rara en el Perú, solo en una ocasión han sido observados en las islas Lobos de Afuera 4 especímenes, se capturó uno de ellos.

Distribución: De México a Panamá, incluyendo todas las islas Oceánicas (Allen y Robertson, 1994). Amplia su distribución hasta las islas Lobos de Afuera, Perú.

FAMilia: TRIPTERYgiIdAe

\section{Axoclinus lucillae Fowler, 1944}

(Fig. 12)

Material examinado: Un espécimen de $19 \mathrm{~mm}$ LS (CZA5), isla Foca, Piura $\left(05^{\circ} 12^{\prime} 11^{\prime \prime} S\right.$; $\left.81^{\circ} 12^{\prime} 35^{\prime \prime} \mathrm{W}\right)$ el 22 de mayo de 1999.

Registros adicionales: También registrado y fotografiado en islas Lobos de Afuera (0656'30"S; 8043'21"W) en marzo de 2000.

Caracteres de diagnostico: $\mathrm{D}$ : $\mathrm{III}+\mathrm{XII}+9$; A: II, 17; P: 15- 16; PP: I, 2; línea lateral gradualmente desciende desde el borde superior del opérculo al eje mediolateral. Base del pedúnculo caudal con una barra blanca perlada, seguida por una barra negro intenso marginado distalmente de rojo (Robertson y Allen 2002).

Observaciones: Especie de pequeño tamaño difícilmente observable. Se le encuentra dentro de pequeñas cuevas rocosas con fondo de conchuela y pequeńas rocas sobre las que reposa. Se encuentran en pequeños grupos, según lo observado, hasta de 6 especímenes.

Distribución: México central a Colombia (Robertson y Allen 2002). Amplia su distribución hasta Isla Foca e islas Lobos de Afuera, Perú.

\section{FAMILIA: GobIIDAE}

\section{Elacatinus puncticulatus (Ginsburg, 1938)}

\author{
(Fig. 13)
}

Material examinado: Dos especímenes de 37 y $41 \mathrm{~mm}$ LS (IMARPE s/n), Canoas de Punta Sal, Tumbes (035'42”S, 


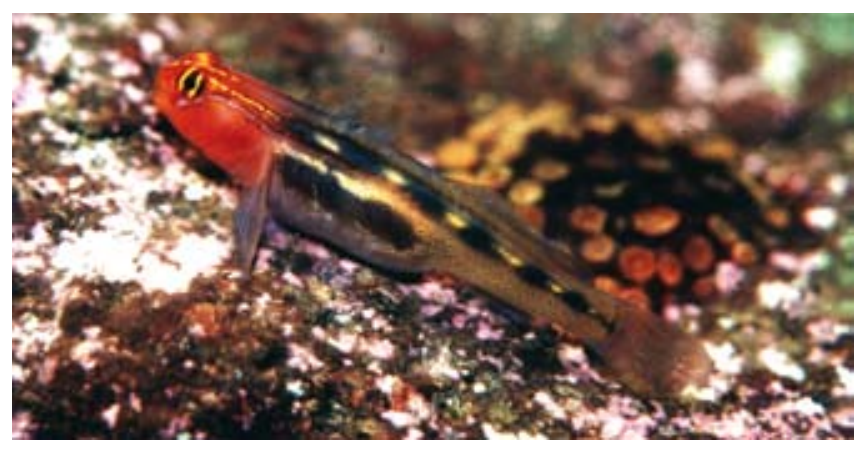

Figura 13. Elacatinus puncticulatus, Canoas de Punta Sal, Tumbes. Noviembre del 2003.

$\left.80^{\circ} 55^{\prime} 01^{\prime} \mathrm{W}\right)$ el 11 de noviembre de 2003. Dos especímenes de 38 y $42 \mathrm{~mm}$ (lote CZA-6), Canoas de Punta Sal, Tumbes $\left(03^{\circ} 55^{\prime} 42^{\prime \prime}\right.$ S, 8055'01"W) el 11 de noviembre de 2003.

Registros adicionales: No se le ha registrado nuevamente.

Caracteres de diagnostico: D VII+I, 11-12); A I, 9-10; P 20-23; disco pélvico presente; cuerpo y cabeza sin escamas; cabeza color rojo con dos líneas detrás de cada ojo, una amarilla y la otra celeste, además de una línea amarilla interorbital; ojo negro, atravesado por dos líneas amarillas verticales; cuerpo amarillento translúcido con 6 a 8 manchas negras sobre una línea dorada en los flancos y una banda negruzca sobre la cavidad abdominal.

Observaciones: Su guarida está conformada por una pequeńa y estrecha grieta en la roca, desde donde sale a alimentarse y patrullar su pequeño territorio. Raro en la zona de registro, pero se observaron varios individuos. Todos los especímenes se encontraron en un solo arrecife rocoso.

Distribución: Del Golfo de California a Ecuador (Robertson y Allen 2002). Amplia su distribución hasta Canoas de Punta Sal, Perú.

\section{Coryphopterus urospilus Ginsburg, 1938}

(Fig. 14)

Material examinado: Dos especímenes de 28 y $31 \mathrm{~mm}$ LS (IMARPE s/n), Bahía Samanco, Ancash (09¹2’04”S; 78³3'27"W) el 6 de agosto de 1997. Un espécimen de $38 \mathrm{~mm}$ LS (IMARPE s/n), Isla Foca, Piura (05¹2’25,2”S, 8112’20,52 W) el 20 de mayo de 1999. Un espécimen de $57 \mathrm{~mm}$ LS (CZA-

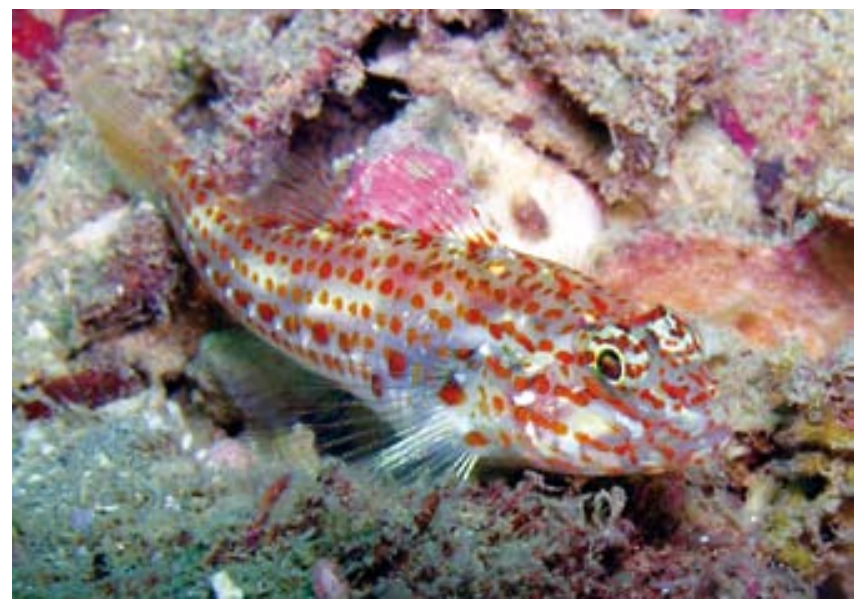

Figura 14. Coryphopterus urospilus. Punta Sal, Tumbes. Octubre del 2007.
7), Punta Sal, Tumbes (03ำ $57^{\prime} 12,5^{\prime \prime}$ S, 8057’49,3”W) el 9 de noviembre de 1999.

Registros adicionales: Es una especie frecuente en Islas Lobos de Afuera y común en las costas rocosas de Tumbes. La fotografía presentada es en Punta Sal, el 16 de octubre del 2007

Caracteres de diagnostico: $\mathrm{D}$ : VI+I, 8-9; A: I, 8-9; P: 19-21; disco pélvico presente; escamas mediolaterales 25-26; escamas grandes, ásperas, en todo el cuerpo, cabeza sin escamas (Robertson y Allen 2002). Cuerpo semi transparente con 5 ó 6 filas horizontales de puntos anaranjados que se inician en la cabeza; 2 líneas de tenues puntos blancos, una sobre el dorso y otra a lo largo de la línea media lateral del cuerpo; tres líneas punteadas anaranjadas sobre las mejillas, la superior atraviesa el ojo; ojo blanquizco con múltiples puntos anaranjados.

Observaciones: Especie común en las costas rocosas de Tumbes, frecuente en Lobos de Afuera. Habita en lugares de aguas tranquilas, en la parte baja de arrecifes rocosos, preferentemente en grietas con piso de arena. Se mueve sobre la arena de los alrededores apoyado en sus aletas. Su coloración translucida lo hace poco visible sobre la arena.

Distribución: De Baja California, México, a Colombia, incluyendo las islas oceánicas (Galápagos, Isla del Coco, las Revillagigedos y Malpelo). Amplia su distribución hasta Punta Sal, Perú, incluyendo la isla Foca y las islas Lobos de Afuera. Durante El Niño llega hasta bahía Samanco.

\section{Lythrypnus dalli (Gilbert, 1890)}

\section{GuSANito DEL Diablo (Fig. 15)}

Material examinado: Un espécimen de $22 \mathrm{~mm}$ LS (IMARPE s/n), Bahía Samanco, Ancash (09¹2’04”S; 78³3’27”W), 8 de agosto de 1997. Tres especímenes de 20, 24, $25 \mathrm{~mm}$ LS (IMARPE s/n), Isla Foca, Piura $\left(05^{\circ} 12^{\prime} 07^{\prime \prime} S\right.$; 8112'29”W) el 21 de agosto de 1997. Tres especímenes de 23, 15, $28 \mathrm{~mm}$ LS (CZA-8), Punta Sal, Tumbes (0357'12”S, 8057’49”W) el 9 de noviembre de 1999.

Registros adicionales: En enero de 1998, durante El Niño se encontró 2 especímenes juveniles en Pucusana (sector El Chuncho), Lima (12²8'16,7”S, 7647’47,3”W). Además fueron observados y fotografiados en islas Lobos de Afuera donde

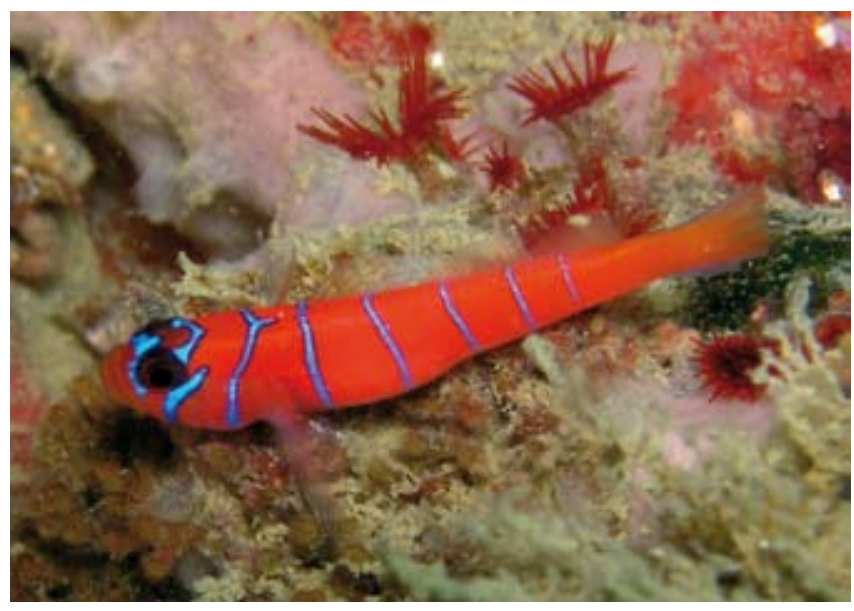

Figura 15. Lythrypnus dalli, Canoas de Punta Sal, Tumbes. Noviembre del 2006. 
son frecuentes. Es una especie común en los arrecifes rocosos de la costa de Tumbes. Después de El Niño no se les ha registrado en isla Foca ni en Pucusana.

Caracteres de diagnostico: D: VI+I, 15-19; A: I, 11-16; P: 17-20. Cabeza con una cresta carnosa longitudinal; disco pélvico presente; cabeza y nuca sin escamas; cuerpo cubierto con escamas ásperas pequeñas; la segunda y tercera espinas dorsales prolongadas en los machos. Color rojo brillante; dos manchas en forma de "V" invertida sobre la cabeza, una por delante de los ojos y la otra postorbital, además de una barra vertical en las mejillas; 4 a 6 barras verticales azules delgadas a los lados del cuerpo; pectoral traslúcida; caudal roja o amarillenta.

Observaciones: Peces territoriales con llamativa coloración de advertencia. Los machos custodian pequeñas grietas donde se ocultan en caso de peligro y para pernoctar y a donde atraen a las hembras posiblemente para desovar. Se les encuentra frecuentemente a más de $10 \mathrm{~m}$ de profundidad en paredes inclinadas de los arrecifes rocosos y dentro de cuevas poco profundas y bien iluminadas.

Distribución: Registrado desde el Golfo de California hasta Ecuador y norte del Perú, incluyendo las islas Malpelo y Galápagos (Allen y Robertson 1994, Chirichigno y Cornejo 2001, Béarez et al., 2007). Se confirma su distribución hasta Punta Sal, Perú, y se incluye las islas Lobos de Afuera. Durante El Niño llega hasta Pucusana, Lima.

\section{Discusión}

Las especies registradas en el presente estudio son un importante aporte al conocimiento de la diversidad marina del Perú, en especial de la costa tropical localizada al norte de Cabo Blanco, en Piura, donde se congrega la mayor riqueza de especies de peces de todo el litoral. De las 275 especies de peces costeros que tenemos registradas en el litoral peruano, incluyendo las 14 reportadas aquí, 198 especies $(72 \%)$ son peces tropicales pertenecientes a la Provincia biogeográfica Panámica, mientras $51(19 \%)$ son típicas de la Corriente Peruana (Provincia biogeográfica Peruana), además de 18 especies que se mueven indistintamente en aguas tropicales y templadas y 8 de origen oceánico que pueden ser encontradas en la costa.

Cabo blanco es el límite natural de distribución sur para la mayoría de los peces de la fauna panámica. En algunos veranos intensos se puede observar una pequeña ampliación de distribución hacia el sur hasta la isla Foca y bahía Sechura, lugares de donde no sobrepasan en condiciones normales, aquí suelen permanecer temporalmente hasta que el invierno se intensifica. Sin embargo, durante el evento El Niño 1997-98 el ingreso de aguas ecuatoriales hacia el sur del Perú permitió que un número importante de peces panámicos se desplazaran hacia el centro y sur del litoral peruano, permaneciendo en localidades refugio más al sur que las mencionadas, hasta finales del verano 1999. Las localidades refugio que consideramos más importante para la detección de indicadores biológicos de aguas tropicales son la Bahía de Samanco, en Ancash, y la bahía de Pucusana en Lima.

Las islas Lobos de Afuera son de particular interés biogeogáfico. Además de los hallazgos que venimos realizando en invertebrados marinos (Hooker et al. 2005, proyecto Esponjas del Perú, Proyecto Equinodermos del Perú), el registro de las especies insulares Prognathodes carlhubbsi y Stegastes beebei podrían ser indicios de un intercambio faunístico entre las islas Galápagos y Lobos de Afuera y reforzaría nuestra teoría de una relación biogeográfica de las islas Lobos de Afuera con el conjunto de islas oceánicas que conforman el Corredor Marino del Pacífico Este Tropical (islas Gorgona, Cocos, Malpelo, Coiba y Galápagos). Un mejor análisis de la información permitirá evaluar la existencia e importancia de estas relaciones faunísticas.

En el litoral somero, además de las especies de interés pesquero, hay un número importante de especies que son difícilmente detectadas. La mayoría de especies reportadas aquí son inconspicuas por su pequeño tamaño, por permanecer ocultas la mayor parte del tiempo o por ser extremadamente raras. La mejor opción para detectar este tipo de fauna es hacerlo por medio de buceo, donde el uso de la fotografía submarina es de gran ayuda pues en ocasiones es casi imposible poder capturar los peces que observamos. La fotografía submarina o la fotografía de especímenes muertos recién capturados, facilita la identificación de algunas especies, como por ejemplo, los del género Stegastes o Thalassoma, cuyas características morfométricas y merísticas se confunden con otras especies próximas, siendo su coloración suficientemente conspicua para reconocer la especie. Sin embargo, en ejemplares juveniles como el colectado de Stegastes beebei, aunque sus características coinciden con las de la especie, la gran similitud morfométrica y merística que presentan los juveniles de las especies del genero no son suficientes para certificar la especie por lo que es recomenbable realizar observaciones en acuario del desarrollo de un juvenil con patrón de coloración similar al presentado hasta que alcance la madurez y se pueda verificar la especie.

Las especies Cirrhitichthys oxycephalus, Lythrypnus dalli y Myripristis leiognathus, fueron citadas para Perú por Chirichigno \& Cornejo (2001). En comunicación personal los autores nos refieren que estas especies fueron incluidas como posibles de encontrar en el norte del Perú, pero sin existir registro objetivo de su presencia, por lo que procedimos a reportarlas según nuestros hallazgos.

\section{Agradecimientos}

Deseo agradecer el gran apoyo de Albertina Kameya y de Juan Vélez en la revisión de nuestros primeros especímenes en los años 1980, cuando aún era estudiante universitario principiante. Agradezco nuevamente a Tina Kameya y al Instituto del Mar del Perú (IMARPE) por su apoyo en los años en que trabajé en dicha institución, tiempo en que se hizo algunos de los hallazgos que aquí se presentan. También deseo agradecer a los doctores Maria Rivera, Abrahán Vaisverg y a los alumnos colaboradores de la Universidad Peruana Cayetano Heredia (UPCH) por su apoyo al área de Biología Marina, al permitir que tanto el laboratorio como la colección científica se vayan consolidando en beneficio de las ciencias marinas del país.

\section{Literatura citada}

Allen G. \& R. Robertson. 1994. Fishes of the tropical eastern Pacific. University of Hawaii Press, Honolulu. 332 p.

Béarez P., 1996. Lista de los peces marinos del Ecuador continental. Rev. Biol. Trop. 44(2):731-741.

Béarez P., J.-T Bujard. \& R. Campoverde. 2007. Description of four small reef fishes from Ecuador: Oxycirrhites typus (Cirrhitidae), Acanthemblemaria balanorum (Chaenopsidae), Arcos decoris (Gobiesocidae) and Lythrypnus dalli (Gobiidae). Cybium 31(4) - pp. 477-479. 
Chirichigno N. 1962. Algunos peces nuevos y poco conocidos de la fauna marina del Perú. Serv. Pesq. Peru 1-29

Chirichigno N. 1963a. Nuevas especies de "Rayas" para la fauna del Perú. Serv. Pesq. Peru 1-13

Chirichigno N. 1963b. Estudio de la Fauna Ictiológica de los Esteros y Partes bajas de los ríos del Departamento de Tumbes (Perú). Ministerio de Agricultura. Sector Pesquería.

Chirichigno N. 1969. Ctenosciaena peruviana nov. sp. Una nueva especie de Sciaenidae de la costa del Perú. Inst. Mar. Lima Ser. Inv. Esp. 18

Chirichigno N. 1973. Nuevas especies de peces de los generos Mustelus (Fam. Triakidae), Raja (Fam. Rajidae) y Schedophilus (Fam. Centrolophidae). Informes Inst. Mar del Peru 1-40

Chirichigno F. N. 1978. Nuevas adiciones a la ictiofauna marina del Perú. Informe $\mathrm{N}^{\circ}$ 46. Instituto del mar del Perú, IMARPE. Callao_Perú. 109 p.

Chirichigno F., N. 1987. Medialuna ancietae nov. sp. Un pez nuevo del mar peruano. Biologia Lima 89-95

Chirichigno F., N. \& T. Iwamoto. 1977. Coryphaenoides delsolari a new species of macrourid fish from the Pacific coast of South America. Proc. Biol. Soc. Wash. 519-528

Chirichigno F., N. \& J. D. McEachran. 1979. Urolophus tumbesensis, a new stingray from the coast of Peru (Batoidea: Urolophidae). Copeia 709-713

Chirichigno N. \& J. Vélez. 1998. Clave para identificar los peces marinos del Perú. Instituto del Mar del Perú. Publicación Especial, Callao, 500 p.

Chirichigno F. N. \& R. M. Cornejo. 2001. Catálogo comentado de los peces marinos del Perú. Instituto del Mar del Perú, IMARPE, Publicación especial. Callao_Perú. 314 p.

Compagno L.J.V. 1999. Checklist of living elasmobranchs. p. 471498. In W.C. Hamlett (ed.) Sharks, skates, and rays: the biology of elasmobranch fishes. John Hopkins University Press, Maryland.

Eschmeyer W.N. (ed.). 1998 Catalog of fishes. Special Publication, California Academy of Sciences, San Francisco. 3 vols. $2905 \mathrm{p}$.
Fischer W., F. Krupp, W. Scheneider, et al. (eds.). 1995. Guia FAO para la identificación de especies para los fines de la pesca. Pacifico centro-oriental. Roma, FAO. Volumen II y III.1199p.

Heemstra P. y J. Randall, 1993. FAO species catalogue. Vol. 16. Groupers of the world (family Serranidae, subfamily Epinephelinae). An annotated and illustrated catalogue of the grouper, rockcod, hind, coral grouper and lyretail species known to date. FAO Fish. Synop. 125(16):382 p.

Hooker Y. 1990. Primer Registro de Johnrandallia nigrirostris, Gill 1862, en el Perú. Boletín de Lima. No 68: 69-71.

Hooker Y. 1993. Zonación de los Peces del Litoral Rocoso en el Área Comprendida entre las Localidades de Cabo Blanco y Los Órganos. Tesis para obtener el Grado de Bachiller en Ciencias Biológicas. Universidad Nacional De Trujillo.

Hooker Y. 2000. Microspathodon dorsalis (Pisces:Pomacentridae) y Prionurus laticlavius (Pisces:Acanthuridae), dos nuevos registros para el mar peruano. Inf. Prog. Inst. Mar Perú. $\mathrm{N}^{\mathrm{o}} 117$

Hooker Y., F. Solís-Marín, M. Lleellish. 2005. Equinodermos de las Islas Lobos de Afuera (Lambayeque, Perú). Rev. peru. biol. 12(1): 77-82

Humann P. \& N. Deloach, 1993. Reef fish identification. Galápagos. New World Publications, Inc., Florida. 267 p.

Meek S. \& S. Hildebrand. 1928. Marine Fishes of Panama. Publs. Field Mus. Nat. Hist. Zool. Ser. 15(2,3): 331-1045 p

Nelson J. 1994. Fishes of the world. Tercera edición. John Wiley \& Sons, Inc., New York. 600 p.

Robertson R. \& G. Allen. 2002. Shorefishes of the Tropical Eastern Pacific: an Information System. CD-ROM. Smithsonian Tropical Research Institute, Balboa, Panama.

Smith W.D. 2006. Dasyatis longa. In: IUCN 2008. 2008 IUCN Red List of Threatened Species. Consultado el 04 April 2009, www.iucnredlist.org

Vildoso A., J. Vélez, N. Chirichigno \& A. Chirinos de Vildoso. 1999. Diversidad de peces marinos del Perú. Bol. Inst. Mar Perú 18(1-2): pp. 49-76 


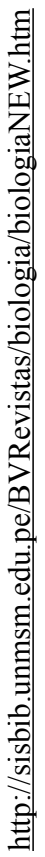

